\title{
DESIGN ANALYSIS OF WIRELESS SENSORS IN BAN FOR STRESS MONITORING OF FIGHTER PILOTS
}

\author{
Goutam Chatterjee',Chaitanya Garg' ${ }^{2}$, Somkuwar . $A^{3}$ \\ ${ }_{1,2}$ Department of Electronics and Communicatin, TCT, Bhopal, India \\ ${ }^{3}$ Department of Electronics and Communicatin, MANIT, Bhopal, India \\ E-mail : 'goutam.chatterjee@gmail.com, ${ }^{2}$ chaitanyagarg.aries@gmail.com, ${ }^{3}$ asomkuwar@gmail.com,
}

\begin{abstract}
It is anticipated that wearable wireless sensors in Body Area Network (BAN) will increase the functionality of lifestyle and health care devices to gradually match the needs of society by year 2010 and people will carry their personal Body Area Network. The applications in areas like lifestyle, assisted living, sports, entertainment functionalities, toxic levels in the lungs of a Fire-Fighter, radiation exposure in war zones etc are already being explored. Yet one very exciting, revolutionary and life saving application in the form of Real time stress monitoring of Fighter Pilots through wearable wireless sensors in BAN has not been thought of. The statistics reveal that $40 \%$ of total flying accidents in IAF occur due to pilot error, however, so far no scientific study has been undertaken to ascertain whether certain non-action/delayed actions taken by pilots in such accidents were related to stress induced. The current paper proposes the entire workable concept which when implemented will be able to provide some valuable insights of human behavior under high ' $g$ ' conditions as well as save lives of pilots.
\end{abstract}

Key words: BAN, Stress, Wireless Sensors, Fighter Pilots, 'g' maneuver, Path-loss Simulation

\section{INTRODUCTION}

Wearable health monitoring system integrated into a telemedical system, implements wireless sensors in Body Area Network. Implanted bio-medical chips have long been matured and were in use since last few decades; however, they are passive chips from which recorded data has to be scanned in close proximity of human or animal body for downloading into an analyzer. It can not facilitate real time continuous monitoring and secondly, only limited medical parameters can be recorded by the implanted biochips.

Tiny wearable wireless sensors are non invasive and can be strapped or stitched into a snug fit jacket for monitoring medical parameters of a human body. When integrated over a communication network it becomes a novel information technology that will be able to support early detection of abnormal conditions and prevention of its serious consequences when fighter pilots are on routine flying missions. Indian Air Force has 39 operational combat squadrons, consisting of approximately 1000 aircraft. The pilots who fly these aircraft are regularly subjected to 4 ' $g$ ' to 8 ' $g$ ' gravitational forces (both negative and positive) for few seconds during every routine sortie. Although, each pilot is trained to handle such high ' $\mathrm{g}$ ' forces and are even imparted "High g centrifuge training", it is a proven medical fact that stress induced due to high ' $\mathrm{g}$ ' maneuver, decreases physiological response, impair decision making capabilities and decrease situational awareness during the short period of time which immediately follows the stress cycle. If an anomaly takes place or emergency occurs precisely during this "impaired period", there is every likely hood that the pilot may not respond quickly enough or does not follow the correct emergency drill.

\section{STRESS MONITORING}

Historically, stress has been defined as a reaction from a calm state to an excited state for the purpose of preserving the integrity of the organism. For an organism as highly developed and independent of the natural environment as socialized man, most stresses are intellectual, emotional and perceptual. There is a distinction between "eustress" and "distress", where eustress is a good stress, such as joy, or a stress leading to an eventful state which is more beneficial to the organism, however here I will refer to stress only as "distress", stress with a negative bias, particularly distress caused by an increase in pilot workload. Therefore, apart from high ' $\mathrm{g}$ ' conditions there is yet another factor to cause stress related anomalies.

In a model for pilot workload, we can identify several types of mental workload tasks that precede each flying mission. This model implies that before a stressor is observed there is an increase in pilot stress due to anticipatory, monitoring and planning effects. In addition, the expected physiological effect of a stressor occurs slightly after the stimulus and may take several seconds to recover. 
IAF losses 20-30 combat aircraft every year in flying accident/incidents. Around $40 \%$ of these are attributed to human error (pilot). Ever if we consider that out of these also, only $10 \%$ were attributed (although there is no scientific study or reasoning) to physiological disorder due to high ' $g$ ' or workload induced stress, then it means that at least one aircraft worth hundreds of crore and an invaluable life is lost every year. This being the motivation factor, current work proposes to conceptualize a telemedical real time stress monitoring system for the fighter pilots, which can avoid the possible loss of aircraft and human life.

The advancement made in miniaturization of wireless sensor and proliferation of Body Area Network makes it possible to cobble together an architecture which can successfully track pilots during flying mission and recall them or instruct them before their reflexes go below the critical level. The entire system will be consisting of:

(a) Wearable micro sensors like EEG, ECG, EMG, respiration, blood pressure, pulse rate, skin conductivity sensor etc. which can be fitted on body hugging fabrics like Lycra made head caps \& inner wears. A compact miniaturized wearable sensor will have three distinct parts viz. transducer, signal conditioning unit and wireless transceiver. It will be powered through tiny Ni-Cd or Li-ion rechargeable cells. The entire structure will be enclosed in an acrylic capsule so that the sensor is safe from body moisture \& heat.

(b) A middleware known as Mobile Base Unit (MBU) will be receiving data from all the sensors wirelessly and then communicate them to a remote server. The MBU can be a PC, PDA or an embedded processor along with wireless transceiver which can be installed anywhere in the airframe of the aircraft.

(c) A remote server with diagnostic software installed at Base Operations Center will communicate with MBU wirelessly again.

To make the concept simpler, the entire system is broken down in different segments and therefore, each part has been analyzed separately in succeeding paragraphs.

\section{BODY AREA NETWORK (BAN)}

Body Area Network (BAN), as far as the class of communication is concerned, have been derived from the concept of wireless local area network (WLAN). Since, the communication nodes are placed on the human body or close to the human body, the term BAN was first coined at Massachuats Institute of Technology \& Science, USA during the year 2002 by the researches.
BAN elements are wireless sensor nodes which have property of low power and non-invasive i.e. not implanted in the human body. These nodes collect physiological data from the human body which needs to be sent to a remote server where the data is processed and analyzed for further course of action. The data collected by sensors are sent via the MBU, which can be located within few meters, to the remote server.

Therefore, the entire communication of data can be subdivided into what we call as "Intra-BAN" and "Extra BAN" communication. While the Intra-BAN communication deals with transfer of data between the wireless sensors and the MBU, the Extra-BAN communication handles the data between the MBU and the remote server. The transmission strategies for both have to be different due to different power levels and different distances involved.

\section{INTRA-BAN COMMUNICATION}

Keeping in mind radiation effects on human body, the power level of sensor nodes will be 1 milli watts or less which will be transmitted through embedded strip or patch antenna. Each node will communicate in broadcast fashion to each other and to the MBU. No external antenna will be used and thus the problem of directivity does not arise. A single channel will be shared by all sensors in contention mode as standardized by IEEE 802.11 standard. Wireless transceivers will be Bluetooth, Zig-bee or UWB (Ultra Wide Band) devices.

In the specific case of the fighter pilot, the total available area or zone where all the sensors and pickups will be deployed; will be monitored while he is in the cockpit. The condition of his body remains in semi crouch while hands are extended towards various operating gadgets of the aircraft like throttle, joystick, controlling levers of armament panel, controlling levers of navigation panel etc.

Therefore, the total body height may be limited to maximum $120 \mathrm{~cm}$ (from floor to the cockpit glass bubble) and $60 \mathrm{~cm}$ in front while hands are fully extended towards the operating panels of the aircraft. If we consider that no sensor will be deployed below knee and also that there would be at least $6 \mathrm{~cm}$ clearance between the cockpit glass bubble and pilot's head, height of sensor zone reduces to approximately $90 \mathrm{~cm}$. Having decided the total zone, where BAN sensor would be operating, to be within the volume of $90 \mathrm{~cm} \times 60 \mathrm{~cm}$, following main input variables need to be considered for successful implementation of BAN communication:-

(a) Number of Nodes. This is a very important variable to be treated in the scenario, as it determines the density of the network. 
(b) Topology. It defines the way the different nodes are deployed as well as the distance among them.

(c) Power Threshold. It indicates maximum loss in a connection among nodes.

(d) Type of Network. Ad-hoc, which means all nodes connecting each other, and infrastructure with all nodes connecting through Access Point (AP), which in this case is the middleware (embedded processor, PCor a PDA)

\section{REQUIREMENT OF SIMULATION}

All the input variables introduced above need to be introduced for the simulation purpose, to MATLAB function. The simulation is need to be run and result of it namely the output variables will be observed, treated and analyzed with the proper tools. In this case the output variables will be:-

(a) Connectivity. Considered as the number of succeeded connections among nodes divided by the number of total connections established in the BAN.

(b) Minimum Link Power. Referred as the minimum power between two links.

MATLAB 7 programming language is chosen to implement a programme which can provide a scenario to be used for studying the behavior of a BAN. This scenario models a fighter pilot's body in the aircraft cockpit in which different nodes of the BAN are deployed. A set of simulations have to be run in order to study the interactions among the nodes that are a part of this BAN. The simulation will obtain the different values of the most important variables being an active part in a BAN and this will lead us to choose best MAC strategy.

Model Set-up. The main source to implement the scenario has been the research work by Philippe De Doncker and Jean Michel Dricot. These researchers have made a series of different simulation experiments in order to model the EM Field propagation around the human body. For these simulations, planes separated $4 \mathrm{~cm}$ along the z-axis of the human torso were taken and placed transmitters on the front of the torso. Then the electric field was observed in the $x-y$ plane at various positions separated by $4 \mathrm{~cm}$ around the human torso. 570 data points were taken at different positions and found that the channel parameters changed depending upon the position around the human body. This effect was modeled in a "Path Loss Model" where path loss depends on a series of parameters such as:-

$n=$ Path loss exponent,$\quad d=$ Distance from the antenna,$\quad s=$ Shadow deviation d Reference distance $\quad P=P$ ath loss at the reference distance
Path loss is given by an empirical power decay law which adds statistical functions of the signal by the means of a zero-mean Gaussian variable:-

$\mathrm{P}_{\mathrm{db}}=\mathrm{P}_{\mathrm{odb}}+10 \cdot \mathrm{n} \cdot \log \left(\frac{d}{d o}\right)+\mathrm{N}(0, \sigma)$

This equation for path loss will be adopted in order to model the path loss for the fighter pilot in aircraft cockpit. For the full MATLAB scenario implementation, further specifications are the following ones:-

(a) Firstly it is assumed that all the pickups and sensors worn by the pilot are visible to each other by placing them on head, chest, shoulders, arms and up to thigh on the legs. If we visualize the three-D effect of sensor placement only in $x$-y plane then we can conclude that all sensors \& actuators are on the lateral surface of a hypothetical cylinder which encompasses the pilot's body, including his outstretched hands.

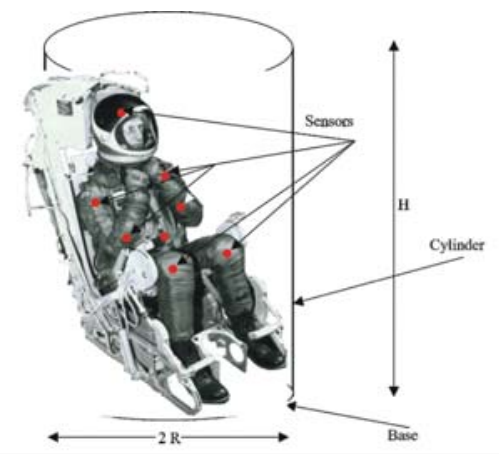

Fig. 1: 3-D Cylinder Encompasses Pilot's Body

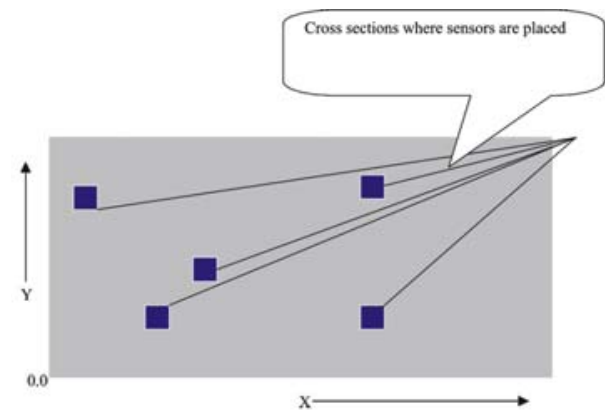

Fig. 2: Lateral Surface of Cylinder in X-Y Plane (Pilot's Body)

If so, then it means that the cylinder has two bases corresponding to both circles with a surface of $\pi R^{2}$ with $R=$ radius of the circle and has a lateral surface given by $2 \pi R$ $\mathrm{H}$ where $\mathrm{H}=$ height of the cylinder. We have taken total distance of outstretched hands from the body to be $60 \mathrm{~cm}$, hence, $2 \mathrm{R}=60 \mathrm{~cm}$.

Since, we are considering only the lateral surface where sensors are placed, the pilot's body will be modeled as rectangle with a height given by $\mathrm{H}=90 \mathrm{~cm}$ and a length 
given by the length of circumference:-

$L=2 \pi R$

By doing so, equation- (1) can be applied for modeling the path loss, because it depends on longitudinal distances and no angular distances have to be taken account.

Since, the cockpit area of a fighter aircraft will restrict a pilot's body to around $90 \mathrm{~cm} \times 180 \mathrm{~cm}(2 \pi \mathrm{R})$, these will be the values used for model. In this rectangle the origin of coordinates is taken at the leftmost and lower vertex.

The next issue for the model refers to the choice of what the position of the different nodes would be. It is proposed that a web may be drawn inside the rectangle by creating cells of $10 \mathrm{~cm} \times 10 \mathrm{~cm}$ each and placing the nodes on cross section of each horizontal and vertical line, drawn to create cells. As a result of it, the different nodes can be found at the coordinates. These are the $x$ and $y$ coordinates of all the possible nodes and therefore there are 171 possible nodes for given dimension of aircraft cockpit.

$$
\left(\frac{L}{10}\right) x\left(\frac{H}{10}+1\right)=\left(\frac{90}{10}\right) \times\left(\frac{180}{10}+1\right)=9 \times 19=171
$$

The leftmost and the rightmost nodes in the rectangle are the same nodes due to the fact that the lateral surface is continuous and so the coordinate $x=0$ is also coordinate $x=180$. Thus the actual possible nodes reduce from 171 to 164.

Therefore, so far we have defined input variables, output variables and the possible place for the different nodes of the BAN.

\section{MATLAB SIMULATION: CONDITIONS \& DEFINITIONS}

The first simulation which was carried out, was very basic of a scenario with BAN, which means that no traffic was generated, frequency was not considered and nor the variation of time. The only factor which characterizes the model-simulation is distance. Therefore, the basis for the connection and connectivity is the eqn-(1), which models the path loss in an established communication among nodes and is depending on the distance parameter.

The starting point was the rectangle of 164 possible nodes where rectangle length is defined as $N_{x}=180$, height $N_{y}=60$ and separation among nodes are:-

Delta $\mathrm{x}_{\mathrm{x}}=10$ and Delta $_{\mathrm{y}}=10$

The of nodes are an input variable and can be set at any value up to 164 but since BAN is not formed by a big amount of nodes but for a precise and chosen amount of them. Therefore, we decided that nodes should not be less than 5 and not more than 35 . Number of nodes is used to generate two different series of random arrays which give us a random set of source (transmitter) and destination (receiver) nodes among the possible coordinates in the table-1. Both the array size are 20 nodes each so as to cover maximum of 35 nodes of BAN. As a result of random generation of different nodes, there is possibility that some of the source and destination nodes are repeated in arrays. Once the set of source and destination nodes are generated, it is necessary to calculate the path loss among the different source and destination nodes with the help of eqn-(1). As this equation is function of path loss vs. distance, distance among nodes must be calculated. This is done by subtracting coordinates of the source and destination nodes. This will yield the difference in $X$ and $Y$ coordinates which will be used to calculate the distance among nodes.

As a first simulation attempt, we only subtracted first source node with first destination node, second source node with second destination node and so on. It means that distances from every source node to every destination node is not considered but only a number of them. Distance ' $d$ ' among source \& destination node is given by:-

$\mathrm{d}=\sqrt{\left(\text { diff_in_ } \mathrm{x} \_ \text {coord }\right)^{2}+(\text { diff_in_y_coord })^{2}}$

Once the distance (d) values are obtained, it is possible to apply eqn-(1) to get the different path loss among different source and destination nodes. At this point of simulation we added two more input variables namely, transmitted power (output power of sensor node) and sensitivity (of destination node) to obtain one very important parameter that is connectivity. Since, BAN transmission levels have to be restricted due to limited battery power and human endurance to EM exposure, normal sensors should transmit $1 \mathrm{~mW}(0 \mathrm{dBm})$ but for simulation purpose we varied transmitted power between $\Delta 10 \mathrm{dBm}$ to $+10 \mathrm{dBm}$. Since, we can expect sensitivity of receivers of the order of $\Delta 85 \mathrm{dBm}$; sensitivity can be varied between $\Delta 65 \mathrm{dBm}$ to $\Delta 100 \mathrm{dBm}$.

Path loss subtracted from $P_{\text {trans }}$ will be the received power $P_{0}$ at destination node and if this $P_{0}$ is greater than receiver sensitivity, we obtain connectivity as the output variable.

Connectivity is defined as the number of succeeded connections divided by the number of total connections. Number of total connections is given by the pair of source node- destination node attempting to communicate. 


\section{SIMULATION RESULTS}

Random arrays of source nodes as orange lines and destination nodes as magenta lines, selected by the simulation model are displayed in 3-dimension in Fig-3. The simulation model thus, could clearly identify the source nodes and the destination nodes.

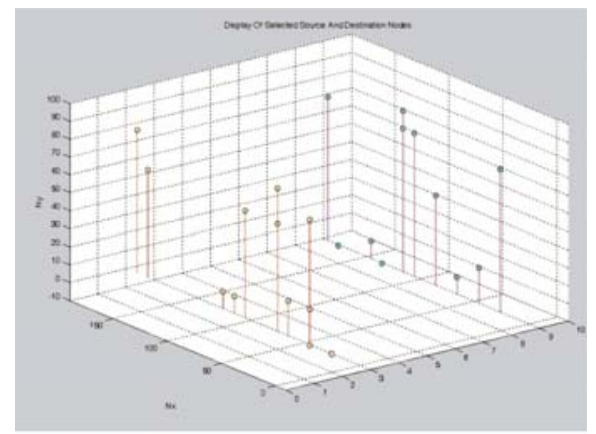

Fig. 3 Display of Selected Source and Destination Nodes

Connectivity as a function of transmitted power and receiver sensitivity with variation in the values of both, is displayed in Fig-4. Use of GUI could enable us to vary transmitted power as well as the receiver sensitivity and accordingly a different set of connectivity could be observed.

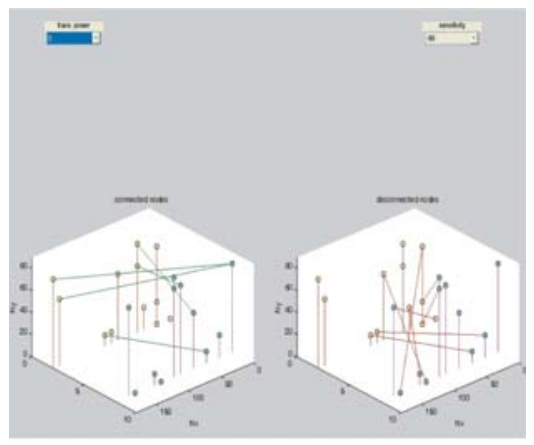

Fig. 4 Connectivity Among Source and Destination Nodes Using GUI

\section{EXTRA-BAN COMMUNICATION}

Two possible approaches are there to establish extra BAN communication between the MBU and the remote server on the ground:-

1. All fighter aircraft have UHF and VHF voice channels for communicating air to air and air to ground. Due to available aircraft height above the ground surface, ranges up to $300 \mathrm{Km}$ are normal. Each airborne and ground trans-receiver has capability to handle 8000 voice channels. Any one of the VHF/UHF channel can be utilized to modulate pilot's physical condition data for transmission to ground server. The only limitation in this approach is when aircraft flies beyond the radius of $300 \mathrm{Km}$ or when it carries out low level flying behind terrain obstacles, transfer of data will not be continuous to the server and at times the connection will be broken for the significant period of flying.

2. The second approach also utilizes the VHF/UHF channels but in this case a double hop communication is established first by transmitting the data to an AWAC (Airborne Warning and Control) aircraft or a stand-off Jammer aircraft or a refueler aircraft, which is generally at a greater height and away from the actual fighter formation aircraft. Data will than be relayed by stand-off aircraft to the ground server. The second hop can be transmitted utilizing VHF/UHF channel or a satellite link which is possible through an AWAC class of aircraft.

Extra-BAN channels will have no power restrictions and thus can be reliably established. Security of data can be ensured by encrypting the data link.

\section{GROUND SERVER, DIAGNOSTIC \& APPLICATION}

Ground server can be established in the Base Operations Center with backup server and data warehousing at another remote location either at the same airbase or at command $\mathrm{HQ}$. The server should have following capabilities:-

(a) Medical parameters of all the pilots flying out of the base station and visiting pilots from other flying stations. The parameters should include data under different physical conditions along with known symptoms or limitations.

(b) Each pilot needs to be identified through a unique code or voice recognition software programme.

(c) It should be able to set different threshold levels for each medical parameter for each pilot.

(d) Creation of Alerts as and when the received data goes beyond threshold. Alerts should be recorded along with the occurred medical condition i.e. ECG, EEG, BP data at the time of alert generation.

(e) Generation of report after each flying sortie for each pilot so that individual weaknesses can be identified

Based on above stated capabilities, diagnostic software has to be developed to run on the server.

\section{CONCLUSION}

A broad frame work for the stress monitoring of fighter pilots in real time wireless sensors has been prepared. Since Extra-BAN communication would take place on an already tried, tested and established communication network, our much of the work is being focused on Intra- 
BAN communication. Currently, we are carrying out simulations on MATLAB environment to establish various communication parameters.

The second version of simulation is currently under progress with following deviations:-

(a) Instead of multi hop, single hop is chosen.

(b) Instead of source nodes and destination nodes, only active nodes are chosen.

(c) Instead of first source node communicating with first destination node, every active node communicates with all other nodes.

(d) Two new inputs, topology and infrastructure is chosen.

(e) Two new output variables, mean power and power variance are introduced.

The objective of second version of simulation is to arrive at a more robust communication protocol to sustain connectivity in BAN

\section{REFERENCES}

[1] C Otto, A Milenkowik, et al, 2006, "System Architecture of a wireless Body Area Sensor Network for Ubiquitous Health Monitoring", Journal of Mobile Multimedia, Vol. 1, No(4), pp307-326.

[2] E Jovanov, A O Lords, et al, May/June 2003, "Stress Monitoring using a Distributed Wireless Intelligent Sensor System", IEEE engineering in Medicine and Biology Magazine".

[3] D Konstantas, A V Halteren, et al, 2-4 June 2004, "Mobile Patient Monitoring: The Mobihealth system", international Congress on Medical and Care Compunetics, NCC, The Hague.

[4] P D Doncker, et al, 2005, 'Ultra Wide-Band Body Area Channel Model', International workshop on wireless Ad-Hoc networks, London, UK.

[5] J N Dricot, et al, 2005, 'Multivariate Analysis of the cross layer Interaction in wireless networks Simulation', International workshop on wireless Ad-Hoc networks, London, UK.

[6] C L Barrett and Drozda M, June 2004, 'Analyzing interaction between network protocols, topology and traffic in wireless radio networks', IEEE magazine of wireless communication, pp-36-37.

[7] I E Lamprinos, et al, Sep. 2004, 'A low power Medium Access Control protocol for wireless medical sensor networks', IEEE Bio Medical publications, pp23-26.
[8] 'Statistical Toolbox to use with MATLAB. User's guide version5', MATLAB release 14, May2004.

[9] Andrew S Tannenbaum, 2003 'Computer Networks. 4th Edition,

[10] Ilker Demirkol, et al, June 2003 'MAC protocols for wireless sensor Networks: A Survey', IEEE transactions on Network \& service management.

[11] Department of Acceleration Physiology, Institute of Aerospace Medicine, Indian Air Force, Bangalore, Ind J Aerospace Med 50 (1), 2006.

[12] M J Skinner, P A Simpson, 2002, "Workload issues in military tactical aircraft", Int J of Aviation, Psych., vol 12, no. 1, pp 79-93.

[13] MIG crash Chronology, www.defenceindia.com.

[14] Indian Air Force aircraft, www.defenceindia.com

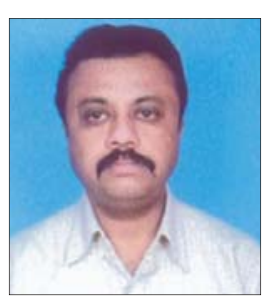

Goutam Chatterjee has received B.E in E\&TC from JEC Jabalpur and M.Tech from IIT $\Delta$ Kharagpur in the year of 1981 and 1991 respectively. Currently he is pursuing his Phd at MANIT-Bhopal. He is working as Professor and Vice Principal in Thakral College of Technology, Bhopal. He also has 27 years of varied experience in defense, private sector and academic field.

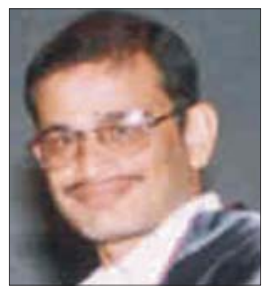

Dr. Ajay Somkuwar has received E\&TC B.E. from JEC, Jabalpur and M.Tech. from MACT, Bhopal in the year 1991 and 1994 respectively . Also he received Ph.D. from IIT, Delhi in 2003.He is an expert in sensor applications and till date has guided numerous projects in the field. His research work has published in many national and International symposiums. Currently as Asst Professor in EC department of MANIT, Bhopal; is supervising $04 \mathrm{Ph} . \mathrm{D}$. research works. 